\title{
EFICIÊNCIA BIOLÓGICA DE GLYPHOSATE NO CONTROLE (DESSECAÇÃO) DO CAPIM-ANDROPOGO
}

\author{
Antônio Carlos de Barros ${ }^{1}$ e Márcio J. Scaléa ${ }^{2}$
}

'EMATER-GO. Caixa Postal 211. Jataí, GO 75800-012

${ }^{2}$ MONSANTO Agrícola. Rua Paes Leme, 524. São Paulo, SP 05424-904

\begin{abstract}
RESUMO
Foi conduzido um ensaio no município de Jataí-GO, com objetivo de avaliar a eficiência biológica de glyphosate em duas formulações, com ou na ausência de adjuvantes à calda de pulverização, para o controle (dessecação) do capim-andropogo (Andropogon gayanus). O delineamento experimental adotado foi o de blocos ao acaso, com quatro repetições e quinze tratamentos. Os tratamentos químicos utilizados, com as respectivas doses do equivalente ácido foram: glyphosate na formulação grânulos auto dispersíveis em água (WG) a 1080, 1440, 1800 e $2160 \mathrm{~g} / \mathrm{ha}$; glyphosate, na formulação concentrado solúvel (CS), a 1080, 1440, 1800 e $2160 \mathrm{~g} / \mathrm{ha}$; glyphosate, nas duas formulações, a $1800 \mathrm{~g} / \mathrm{ha}$; com a adição à calda de pulverização dos adjuvantes SILWET L-77- Ag 0,05\% v/v, ou com ASSIST 0,3\% v/v ou ainda com óleo diesel $0,3 \% \mathrm{v} / \mathrm{v}$, além da testemunha. O herbicida dessecante foi aplicado em 12/01/99, quando a gramínea encontrava-se em desenvolvimento vegetativo intenso, com a altura de 30 a $40 \mathrm{~cm}$, não apresentando nenhum tipo de estresse. Verificou-se que o glyphosate a 1800 ou $2160 \mathrm{~g} / \mathrm{ha}$, nas formulações WG e CS, glyphosate a $1800 \mathrm{~g} / \mathrm{ha}$, independentemente da formulação, com a adição à calda de pulverização de um dos adjuvantes SILWET L-77 Ag ou ASSIST ou ainda óleo diesel, propiciou eficiente controle (dessecação) do capim-andropogo. Pode-se recomendar glyphosate, independentemente da formulação utilizada, em doses iguais ou superiores a $1800 \mathrm{~g} / \mathrm{ha}$, com ou na ausência de adjuvantes, para o controle do capim-andropogo, em condições favoráveis do ambiente. A adição de adjuvantes a solução contendo glyphosate não propiciou aumento significativo no controle dessa gramínea.
\end{abstract}

Palavras-chave: Andropogon gayanus, adjuvantes, plantio direto, integração agropecuária.

\section{ABSTRACT \\ Glyphosate biological efficacy in the control of andropogo grass}

Andropogo grass (Andropogon gayanus) is a forage very well adapted to poor pasture soils throughout Cerrados (Brazilian savannas). During the last years, with the expansion of no tillage, specially within the cropping system named "crop/pasture integration", where glyphosate is the main tool the farmers have to clean the fields before planting. Andropogo grass became a problem weed due to its natural resistance to that herbicide. Andropogo leaves are strongly hairy, and this is, probably, the cause of that resistance. The field trial was carried out in Jataí-GO, to evaluate the efficacy of two glyphosate formulations (Roundup CS-liquid and Roundup WG-soluble granules), alone and in tank mixtures with three adjuvants (Silwett L77, Assist and diesel oil) in the postemergence control (burndown) of andropogo grass. Adjuvants were supposed to improve glyphosate uptake, overcoming the presence of hairs on the leaf surface. Results showed that: 1- there were no differences between the two glyphosate formulations; 2- the addition of adjuvants slightly improved efficacy, but differences were not statistically significant; 3- Andropogo control can be obtained by the use of glyphosate at the rate of, at least, 1800 $\mathrm{g} \mathrm{AE} / \mathrm{ha}$, with or without adjuvants, when the grass is under an intense vegetative growth, with 30 to $40 \mathrm{~cm}$ of height.

Key Words : Andropogon gayanus, adjuvants, no tillage, crop/pasture integration. 


\section{INTRODUÇÃO}

O sistema de plantio direto na palha ocupa extensas áreas no Brasil. No entanto, segundo Leite (1995), alguns problemas são encontrados na adoção do sistema e entre eles está o controle adequado da vegetação daninha, assegurando em grande parte, o sucesso da cultura instalada nesse sistema. Já no Brasil Central, em conseqüência da dificuldade de cultivo durante a entresafra, em função principalmente do deficit hídrico, são poucas as alternativas de culturas que apresentam potencial para a produção e ou formação de cobertura morta, visando o plantio direto das culturas de verão (Pereira, 1995). Assim, espécies como o capim andropogo (Andropogon gayanus), eventualmente no ecossistema cerrado, tem sido utilizado como cobertura vegetal para a implantação do plantio direto na palha, em especial nas propriedades onde se pratica a integração agricultura e pecuária.

O capim-andropogo é uma planta perene, com grande capacidade de perfilhamento, bastante rústica, adaptando em solos com baixa fertilidade, podendo atingir até três metros de altura, folhas com intensa pilosidade, retendo orvalho praticamente durante quase todo o período da manhã.

O controle químico dessa espécie, por meio de herbicidas em pós-emergência (dessecantes), tem sido deficiente, mesmo utilizando doses elevadas de glyphosate, isso, possivelmente, é ocasionado pela intensa pilosidade do limbo foliar da referida gramínea, que pode dificultar a absorção do herbicida. Entretanto, o manejo mecânico tem sido a opção mais viável para o controle dessa espécie (Carvalho, 1995).

Por sua vez, para aumentar a eficiência biológica de muitos herbicidas de aplicação em pós-emergência tem sido utilizado adjuvantes à calda de pulverização, o que pode representar economia na aplicação, pela possibilidade do uso de doses reduzidas do herbicida (Sherrick et al., 1986, citado por Vargas et al., 1997).

O objetivo desse trabalho foi o de avaliar a eficiência agronômica do herbicida glyphosate aplicado em pós-emergência, isolado ou com a adição de adjuvantes à calda de pulverização, no controle (dessecação) do capim-andropogo.

\section{MATERIAL E MÉTODOS}

O experimento foi instalado na Fazenda Campo Redondo, localizada no município de Jataí-GO, região fisiográfica Serra do Caiapó. O delineamento experimental, adotado foi o de blocos ao acaso, com quatro repetições e quinze tratamentos, relacionados na Tabela 1 .

Tabela 1. Herbicidas utilizados, formulação e doses do produto comercial (p.c.) e do ingrediente ativo (i. a.). Jataí-GO, 1998/99.

\begin{tabular}{|c|c|c|c|c|}
\hline \multicolumn{2}{|c|}{ Tratamento } & \multirow{2}{*}{ Formulação } & \multicolumn{2}{|c|}{ Dose } \\
\hline Produto Comercial & Ingrediente Ativo & & kg ou l/ha (p.c.) & g e.a./ha \\
\hline Roundup & Glyphosate & $\mathrm{WG}^{4}$ & 1,5 & 1080 \\
\hline Roundup & Glyphosate & WG & 2,0 & 1440 \\
\hline Roundup & Glyphosate & WG & 2,5 & 1800 \\
\hline Roundup & Glyphosate & W G & 3,0 & 2160 \\
\hline Roundup & Glyphosate & $\mathrm{CS}^{5}$ & 3,0 & 1080 \\
\hline Roundup & Glyphosate & $\mathrm{CS}$ & 4,0 & 1440 \\
\hline Roundup & Glyphosate & $\mathrm{CS}$ & 5,0 & 1800 \\
\hline Roundup & Glyphosate & $\mathrm{CS}$ & 6,0 & 2160 \\
\hline Roundup ${ }^{1}$ & Glyphosate & $\mathrm{CS}$ & 5,0 & 1800 \\
\hline Roundup ${ }^{1}$ & Glyphosate & WG & 2,5 & 1800 \\
\hline Roundup ${ }^{2}$ & Glyphosate & $\mathrm{CS}$ & 5,0 & 1800 \\
\hline Roundup ${ }^{2}$ & Glyphosate & WG & 2,5 & 1800 \\
\hline Roundup $^{3}$ & Glyphosate & $\mathrm{CS}$ & 5,0 & 1800 \\
\hline Roundup $^{3}$ & Glyphosate & WG & 2,5 & 1800 \\
\hline Testemunha & Testemunha & - & - & - \\
\hline
\end{tabular}

${ }^{\prime}$ Foi adicionado a calda de pulverização o adjuvante SILWET L-77 Ag a 0,05\% v/v

${ }^{2}$ Foi adicionado a calda de pulverização o adjuvante ASSIST $0,3 \% \mathrm{v} / \mathrm{v}$

${ }^{3}$ Foi adicionado a calda de pulverização óleo diesel a $0,3 \% \mathrm{v} / \mathrm{v}$

${ }^{4} \mathrm{WG}$ - Grânulos auto-dispersíveis em água

${ }^{5} \mathrm{CS}$-Concentrado solúvel 
O capim-andropogo, foi pastejado, retirando-se os animais para permitir a rebrota dessa espécie e 45 dias após foi efetuada a aplicação dos tratamentos, quando essa gramínea encontrava-se de 30 a $40 \mathrm{~cm}$ de altura, com bastante área foliar, pois, apresentava-se em pleno vigor vegetativo, cobrindo $95 \%$ do solo.

O herbicida foi aplicado no dia 12/01/99, das 10:15 h às $11: 20 \mathrm{~h}$, após a evaporação quase total do orvalho retido nos limbos dessa gramínea, tendo no final da aplicação, temperatura do ar $28^{\circ} \mathrm{C}$ e umidade relativa de $75 \%$.

As parcelas experimentais mediram 7,0 $\mathrm{m} \times 3,0 \mathrm{~m}=$ $21,0 \mathrm{~m}^{2}$, enquanto que, a área útil para observações mediu $5,0 \mathrm{~m} \times 1,5 \mathrm{~m}=7,5 \mathrm{~m}^{2}$.

O equipamento utilizado na aplicação dos produtos, foi um pulverizador costal pressurizado a $\mathrm{CO} 2$, pontas de pulverização (bicos de jato plano), série 110.02, calibrados para $3,0 \mathrm{~kg} / \mathrm{cm}^{2}$ de pressão, proporcionando um volume de aplicação de 200 litros de calda por hectare.

As precipitações pluviais diárias, no período de dezembro/98 a fevereiro/99, registradas pelo Centro de Ciências Agrárias da UFG, em Jataí-GO, encontram-se na Tabela 2.

As avaliações de eficiência biológica foram realizadas aos 11, 25 e 45 dias após a aplicação dos tratamentos (DAT), adotando-se a escala percentual, sendo que 0 (zero) correspondeu a nenhum controle e 100 (cem) representou controle total das plantas daninhas.

Os resultados de eficiência biológica foram submetidos a análise de variância pelo teste $\mathrm{F}$ e as médias comparadas pelo teste de Tukey a 5\% de probabilidade.

\section{RESULTADOS E DISCUSSÃO}

Os resultados obtidos para o controle (dessecação) do capim-andropogo, encontram-se relacionados na Tabela 3, Verifica-se que na primeira avaliação de controle dessa gramínea (11 DAT), os tratamentos químicos atingiram níveis entre 72,5 e $88,5 \%$. No entanto, glyphosate WG a 1800 e $2160 \mathrm{~g} / \mathrm{ha}$, glyphosate CS a $2160 \mathrm{~g} / \mathrm{ha}$, proporcionaram níveis de controle equivalentes aqueles obtidos por glyphosate $1800 \mathrm{~g} / \mathrm{ha}$, nas duas formulações, com a adição à calda de pulverização de SILWET L-77 Ag ou ASSIST ou ainda óleo diesel. Já aos 25 e 45 DAT foram observados crescentes índices de controle nos tratamentos químicos, mesmo quando foi utilizado glyphosate nas menores doses.

Aos 45 DAT, verificou-se que o capim-andropogo foi eficientemente controlado pelo herbicida glyphosate, nas formulações WG ou CS, a 1800 e 2160 g/ha, equivalendo-se aos resultados conseguidos por glyphosate, nas formulações
WG ou CS, a $1800 \mathrm{~g} / \mathrm{ha}$, com a adição de adjuvantes à calda de pulverização, o que discorda dos resultados obtidos por Carvalho (1995). Esse autor verificou que a aplicação de glyphosate ou sulfosate, em doses variando de 4,00 a 6,25 1/ha do produto comercial, com ou na ausência de adjuvante, proporcionaram deficiente controle do capim-andropogo.

Tabela 2. Precipitações pluviais diárias, registradas em Jataí-GO, pelo Centro de Ciências Agrárias / UFG. Jataí-GO, 1998/99.

\begin{tabular}{|c|c|c|c|}
\hline \multirow{3}{*}{ Dia } & \multicolumn{3}{|c|}{ Precipitação Pluvial (mm) } \\
\hline & \multirow{2}{*}{$\begin{array}{c}1998 \\
\text { Dezembro }\end{array}$} & \multicolumn{2}{|c|}{1999} \\
\hline & & Janeiro & Fevereiro \\
\hline 1 & 0,8 & 0,1 & 33,3 \\
\hline 2 & 24,6 & 12,7 & 0,0 \\
\hline 3 & 8,1 & 11,2 & 20,4 \\
\hline 4 & 0,1 & 19,9 & 0,0 \\
\hline 5 & 0,2 & 4,0 & 0,0 \\
\hline 6 & 5,6 & 3,9 & 0,0 \\
\hline 7 & 0,0 & 4,6 & 46,4 \\
\hline 8 & 7,0 & 10,8 & 0,0 \\
\hline 9 & 0,0 & 0,0 & 6,0 \\
\hline 10 & 12,3 & 0,0 & 3,0 \\
\hline 11 & 12,4 & 0,0 & 0,0 \\
\hline 12 & 0,3 & 1,2 & 1,2 \\
\hline 13 & 13 & 0,0 & 0,0 \\
\hline 14 & 11,9 & 34,8 & 0,0 \\
\hline 15 & 3,4 & 3,0 & 0,0 \\
\hline 16 & 0,0 & 0,7 & 0,0 \\
\hline 17 & 0,0 & 1,6 & 0,0 \\
\hline 18 & 1,4 & 0,0 & 0,0 \\
\hline 19 & 1,6 & 0,4 & 0,0 \\
\hline 20 & 0,0 & 0,0 & 19,0 \\
\hline 21 & 5,4 & 0,0 & 5.4 \\
\hline 22 & 20,4 & 0,4 & 11,4 \\
\hline 23 & 25,6 & 0,0 & 9,8 \\
\hline 24 & 21,2 & 0,0 & 17,4 \\
\hline 25 & 0,0 & 0,8 & 0,0 \\
\hline 26 & 26,5 & 1,6 & 5,9 \\
\hline 27 & 10,8 & 1,8 & 13,8 \\
\hline 28 & 21,0 & 1,0 & 7,0 \\
\hline 29 & 3,0 & 31,6 & - \\
\hline 30 & 9,1 & 0,0 & - \\
\hline 31 & 0,0 & 31,8 & - \\
\hline TOTAL & 233,0 & 177,9 & 200,0 \\
\hline
\end{tabular}


Tabela 3. Resultados médios de controle de Andropogon gayanus, aos 11, 25 e 45 DAT, por meio do herbicida glyphosate em duas formulações, com e na ausência de adjuvantes. Jataí-GO, 1998/99.

\begin{tabular}{|c|c|c|c|c|c|}
\hline \multirow{3}{*}{ Tratamento } & \multirow{3}{*}{ Formulação } & \multirow{3}{*}{$\begin{array}{c}\text { Dose } \\
\text { (g e.a./ha) }\end{array}$} & \multicolumn{3}{|c|}{ Controle } \\
\hline & & & \multicolumn{3}{|c|}{ Dias Após os Tratamentos } \\
\hline & & & 11 & 25 & 45 \\
\hline Glyphosate & $\mathrm{WG}^{4}$ & 1080 & $75,0 \mathrm{e}^{6}$ & $80,0 \mathrm{~d}^{6}$ & $81,2 d^{6}$ \\
\hline Glyphosate & WG & 1440 & $81,7 \mathrm{~cd}$ & $87,5 \mathrm{bc}$ & $92,5 \mathrm{c}$ \\
\hline Glyphosate & WG & 1800 & 83,7 abcd & $92,5 \mathrm{abc}$ & $96,7 \mathrm{abc}$ \\
\hline Glyphosate & WG & 2160 & $85,7 \mathrm{abc}$ & $94,2 \mathrm{a}$ & $97,7 \mathrm{abc}$ \\
\hline Glyphosate & $\mathrm{CS}^{5}$ & 1080 & $72,5 \mathrm{e}$ & $78,7 \mathrm{~d}$ & $79,7 \mathrm{~d}$ \\
\hline Glyphosate & $\mathrm{CS}$ & 1440 & $80,5 \mathrm{~d}$ & $87,0 \mathrm{c}$ & $93,2 \mathrm{bc}$ \\
\hline Glyphosate & $\mathrm{CS}$ & 1800 & $82,5 \mathrm{bcd}$ & $91,7 \mathrm{abc}$ & $96,0 \mathrm{abc}$ \\
\hline Glyphosate & $\mathrm{CS}$ & 2160 & 84,5 abcd & $93,7 \mathrm{a}$ & $98,0 \mathrm{ab}$ \\
\hline Glyphosate $^{i}$ & $\mathrm{CS}$ & 1800 & $86,0 \mathrm{abc}$ & $93,7 \mathrm{a}$ & $96,7 a b c$ \\
\hline Glyphosate $^{1}$ & WG & 1800 & $86,7 a b c$ & $95,2 \mathrm{a}$ & $96,2 a b c$ \\
\hline Glyphosate $^{2}$ & $\mathrm{CS}$ & 1800 & $85,7 a b c$ & $93,0 \mathrm{ab}$ & $96,2 \mathrm{abc}$ \\
\hline Glyphosate $^{2}$ & WG & 1800 & $86,5 \mathrm{abc}$ & $93,2 \mathrm{ab}$ & $97,2 \mathrm{abc}$ \\
\hline Glyphosate $^{3}$ & $\mathrm{CS}$ & 1800 & $87,2 \mathrm{ab}$ & $96,2 \mathrm{a}$ & $97,2 a b c$ \\
\hline Glyphosate $^{3}$ & WG & 1800 & $88,5 \mathrm{a}$ & $96,7 \mathrm{a}$ & $99,2 \mathrm{a}$ \\
\hline Testemunha & - & - & $0,0 \mathrm{f}$ & $0,0 \mathrm{e}$ & $0,0 \mathrm{e}$ \\
\hline C.V. $(\%)$ & - & - & 2,64 & 2,67 & 2,35 \\
\hline F tratamentos & - & - & $459,40 * *$ & $452,79 * *$ & $585,58 * *$ \\
\hline D.M.S. & - & - & 5,22 & 5,77 & 5,26 \\
\hline \multicolumn{6}{|c|}{ 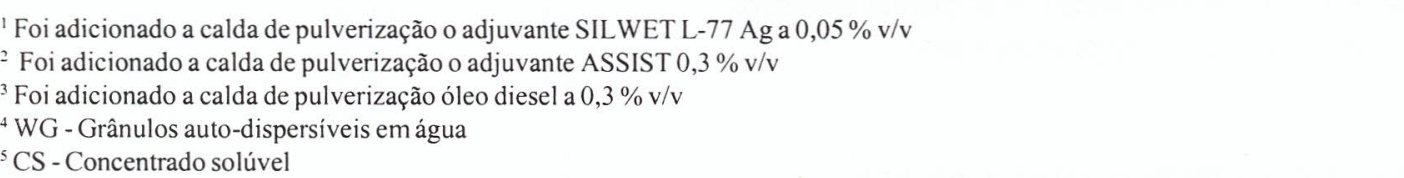 } \\
\hline
\end{tabular}

Observa-se ainda , pela Tabela 3, que a adição de qualquer um dos adjuvantes (SILWET L-77 Ag ou ASSIST ou óleo diesel), à calda contendo glyphosate $1800 \mathrm{~g} / \mathrm{ha}$, nas formulações WG e CS, não propiciou níveis de controle significativamente superiores àqueles conseguidos por glyphosate aplicado isolado, qualquer que seja a formulação do herbicida dessecante. Em outra espécie de difícil controle, o capimcolonião (Panicum maximum), Durigan (1992), observou que a adição de adjuvantes à solução contendo glyphosate proporcionou maiores níveis de controle da referida gramínea, permitindo, com isso, redução das doses de glyphosate. 'De modo geral, houve tendência para maior controle do capimandropogo quando se utilizou glyphosate na formulação WG.

Por outro lado, apesar do glyphosate (1440 g/ha) ter proporcionado índices de controle do capim-andropogo, aos
45 DAT, de 92,5 e 93,2\%, respectivamente para as formulações CS e WG, tais resultados foram considerados insuficientes para essa espécie, pois trata-se de uma planta com capacidade intensa de perfilhamento, porte alto, além de apresentar controle deficiente com os herbicidas aplicados em pós-emergência.

Deve-se considerar que no momento da aplicação do herbicida glyphosate, o capim-andropogo encontrava-se com a altura variando de 30 a $40 \mathrm{~cm}$, portanto, com desenvolvimento vegetativo intenso; isso possivelmente tenha contribuido para o bom desempenho do glyphosate, concordando com Scaléa (1994).

Além disso, as condições do ambiente, no momento da aplicação do herbicida, umidade relativa $75 \%$ e tempera- 
tura do ar $28^{\circ} \mathrm{C}$, foram consideradas favoráveis para a absorção do produto. Isso pode ter favorecido a ação herbicida, fato que pode não ocorrer se a aplicação for efetuada sob condições de estresse (umidade relativa menor que 65\%), concordando com Vargas et al. (1997).

Outro fator que deve ser levado em consideração é a ocorrência de precipitações pluviais, pois em janeiro/99 a intensidade e distribuição das chuvas foram normais na região de Jataí-GO, conforme observa-se na Tabela 2, evitando, com isso, condições de estresse hídrico da gramínea, por ocasião da pulverização do herbicida, contribuindo para boa atuação do glyphosate.

\section{CONCLUSÕES}

Os resultados permitem concluir que não se observaram diferenças significativas entre as formulações WG e CS do glyphosate, no controle do capim-andropogo. Os maiores niveis de controle foram obtidos pelo glyphosate WG a 1800 g e.a./ha, com a adição de óleo diesel $0,3 \%$ v/v, porém, não diferindo significativamente dos outros tratamentos com glyphosate, excetuando nas doses de 1080 e $1440 \mathrm{~g} / \mathrm{ha}$, nas formulações WG e CS, aplicado isolado. A aplicação de glyphosate com aditivos tendeu a proporcionar maiores níveis de controle do capim-andropogo, quando comparada a aplicação isolada do produto. Deve-se utilizar glyphosate em doses iguais ou superiores a $1800 \mathrm{~g} / \mathrm{ha}$, com aditivos ou na ausência dos mesmos, para o controle do capim-andropogo, em condições de desenvolvimento vegetativo intenso (30 a $40 \mathrm{~cm}$ de altura).

\section{LITERATURA CITADA}

CARVALHO, J. A. Controle do capim-andropogo (Andropogon gayanus), com os herbicidas sulfosate e glyphosate. In: ZENECA AGRÍCOLA, São Paulo, SP, ZAPP: O desafio do novo. São Paulo, 1995. p. 107. 109.

DURIGAN, J. C. Efeitos de adjuvantes na calda e do estádio de desenvolvimento das plantas, no controle do capim-colonião (Pannicum maximum) com glyphosate. Planta Daninha, v.10, n. 1/2, p. 39-44, 1992.

LEITE, C. R. I. Eficiência do herbicida ZAPP (sulfosate) sob diferentes condições ambientais, no controle da planta daninha Brachiaria plantaginea (capim-marmelada). In: ZENECA AGRÍCOLA, São Paulo, SP. ZAPP : O desafio do novo. São Paulo, 1995. p. 41-52.

PEREIRA, F. A. R. Determinação da dose eficiente de dessecantes sistêmicos no manejo de Brachiaria decumbens em plantio direto da soja, na região dos cerrados. In: ZENECA AGRÍCOLA, São Paulo, SP. ZAPP : O desafio do novo. São Paulo, 1995. p. 95-99.

SCALÉA, M. J. Plantio direto no cerrado. São Paulo : MONSANTO do Brasil, 1994. 14p.

VARGAS, L.; FLECK, N. G.; CUNHA, M.M. da. ; VIDAL, B.A. Efeito de adjuvantes adicionados à calda herbicida contendo glyphosate. Planta Daninha, v. 15, n.2, p. 206-214. 1997. 
